\title{
The Linac and Booster RF Systems for a Dedicated Injector for SPEAR*
} J.N. Weaver, S. Baird, M. Baltay, M. Borland, $†$ H.-D. Nuhn, J. Safranek, C. Chavis, L. Emery, $\dagger$
R.D. Genin, R. Hettel, H. Morales, J. Sebek, J. Voss, D. Wang, $\ddagger$ H. Wiedemann, B. Youngmann Stanford Sychrotron Radiation Labonatory, Stanford, California 94909 and

R.H. Miller

Stanford Linear Accelerator Center, Stanford, California 94909

SLAC-PUB- 5515

DE91 012376

\section{Abstract}

A $120 \mathrm{MeV}, 2856 \mathrm{MHz}$, TW linac, with a microwave gun, alpha magnet, and chopper, has been built at SSRL as a preinjector for and along with a $3 \mathrm{GeV}$ booster synchrotron ring. The resulting injector will be available on demand to fill SPEAR, which is a storage ring now dedicated to synchrotron light production. The linac sections were purchased from China, the XK-5 klystrons were obtained surplus from SLAC, the modulators are a variation on those at SLAC and were built by SSRL, the alpha magnet and chopper were designed and built at SSRL and the microwave gun was designed and built in collaboration with Varian Associates. The RF system for the booster ring is similar to those at SPEAR and PEP and was built by SSRL. Some of the interesting mechanical and electrical details are discussed and the operating characteristics of the linac and ring RF system are highlighted.

\section{IN TRODUCTION}

The Stanford Positron Electron Accelerator Ring (SPEAR) started out in the early 1970 s as an electron positron high energy physics facility with two colliding beam interaction regions. In 1974 the $\psi-J$ particle was discovered independently at SLAC's SPEAR and BNL's (Brookhaven National Laboratories) AGS. With the advent of PEP and SLC/SLD at SLAC, the high energy physics effort at SPEAR wound down and since 1989 it has been used solely as a source for synchrotron radiation for SSRL and visiting experimenters. Initially, it was thought that SLC could continue to fill SPEAR on some reasonable schedule, but that has not been possible in practice. So a little over three years ago a major project was begun at SSRL to build a dedicated full energy injector. [1] The new injector consists of: 1) a $2 \mathrm{MeV}$ microwave electron gun 2) a $120 \mathrm{MeV}, 9 \mathrm{~m}$ long, short pulse, $2856 \mathrm{MHz}$ linac and 3) a $3 \mathrm{GeV}, 134 \mathrm{~m}$ in circumference, $358.54 \mathrm{MHz}$ synchrotron booster ring, all pulsing at a $10 \mathrm{~Hz}$ rate. The day before Thanksgiving of 1990 a $2.34 \mathrm{GeV}$ beam was injected and stored in SPEAR, marking the end of the formal construction and commissioning phases. Remoting of controls, improving the stability, familiarizing operators with the controls and quantifying machine characteristics has been the thrust of recent efforts. In the following sections a description is given of the injector's two separate and different frequency RF systerns. Synchronization of the two,

\footnotetext{
* Work supported by Department of Energy contract DEAC03-76SF00515 and the Office of Basic Energy Sciences, Division of Material Sciences.

$\dagger$ Now at Argonne National Labs, Argonne, IL 60439.

$\ddagger$ Now at Bates Linac-MIT/LNS, Middleton, MA 01949.
}

non-harmonic (unfortunately) systems is achieved through the linac's chopper. [2] Synchronization of injection into SPEAR is discussed elsewhere. [3]

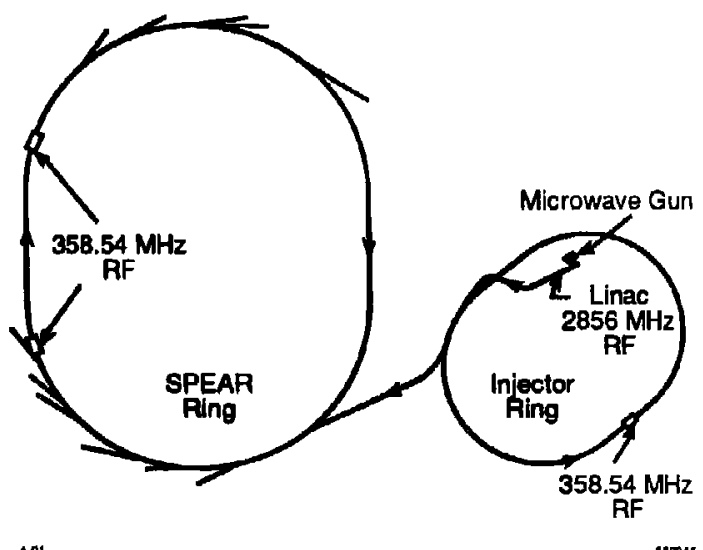

Figure 1. SSRL's Major RF Systems.

\section{I1. The Linac}

The linac, as shown in Fig. 1, injects at a $10 \mathrm{~Hz}$ rate, three, $120 \mathrm{MeV}$ bunches, spaced by $350 \mathrm{ps}$ and of $\approx 4 \times 10^{\circ}$ electrons each, into the injector booster ring through a series of bend magnets, a pulsed kicker magnet and a septum magnet. A $2 \mathrm{MeV}$ microwave gun puts out less than a $2 \mu \mathrm{s}$ long string of several thousand bunches. Since eacl. bunch is the result of a portion of a sinesoidal electric field on a thermonic cathode, the energy spread and longitudinal extent of each bunch is considerable. [4]. An alpha magnet with an internal scraper is used to compress the bunches longitudinally and scrape of a large portion of the low energy tail. Before entering the linac a fast rising pulse, traveling upstream on a stripline chopper, sweeps the multibunch beam by a slit. The result is that only a 1proximately three bunches emerge to be accelerated by the linac. The gun-to-linac layout is shown in Fig. 2. and it is described in detail in references $[2,4,5]$ and $[6]$. Figure 3 shows the linac RF circuit schematically and Fig. 4 shows the high power part of the RF circuit isometrically.

Three, SLAC-type, $2 \pi / 3$ mode, traveling-wave acceleralor sections (DLWG's) were purchased from IHEP (l:1stitute of High Energy Physies), Beijing. PRC. The DLIY'( tuning was checked at SLAC as part of an acceptance test and found to be very good. Furthermore, a high power 


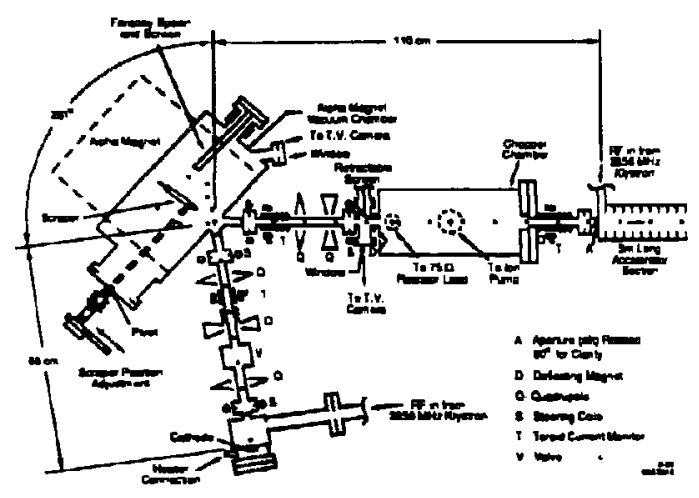

Figure 2. Gun-to-Lianc Layout.

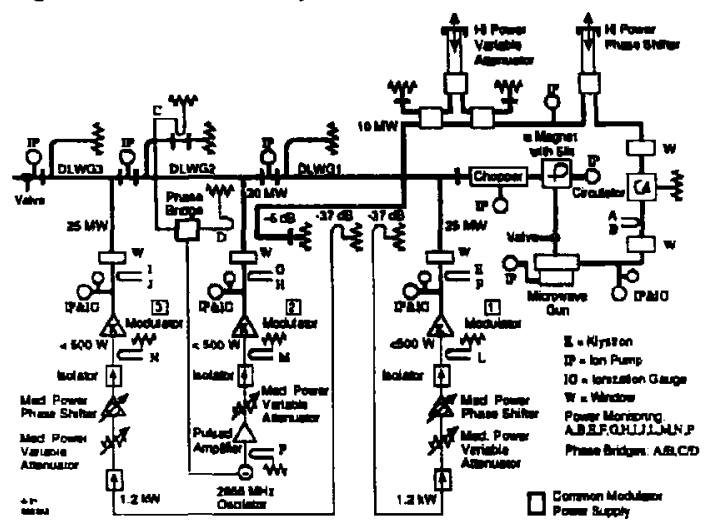

Figure 3. SSRL Injector Linac RF System Schematic.

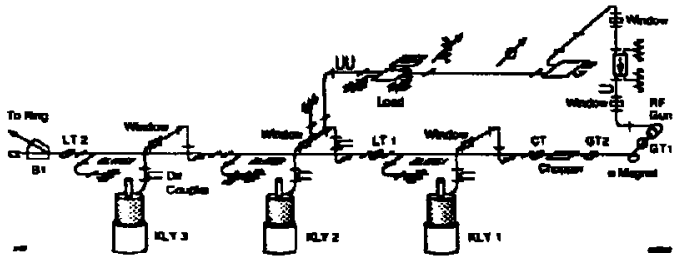

Figure 4. Isometric View of the Linac.

waveguide phase shifter, a high power waveguide attenuator and high power kanthal-coated stainless steel vacuum waveguide loads were purchased from IHEP, which in turn obtained them from domestic PRC industries. External copper cladding and soldered on water lines were added by SSRL to make them water cooled like those at SLAC. A 24 hour, $200^{\circ} \mathrm{C}$, nitrogen gas flushing followed by a 72 hour, $200^{\circ} \mathrm{C}$, vacuum bake preceded installation on the linac. This SLAC baking receipt greatly reduced the water vapor, et cetera, absorbed in the kanthal and thus considerably lessened the time required for RF processing of the linac. The phase shifter and attenuator operate well now that some mechanical drive mechanism problenus las. been attended to.

SLAC fabricated vacuum rectangular waveguide ni.1works connect the three surplus SLAC Xk-5 klysirons t. the linac and the microwave gun. The klystrons are minered by a modified SLAC type modulator. whic!: is fod lis a $480 \mathrm{VAC}, 3 \phi$, variable voltage transformer. Following the transformer is a single, $25 \mathrm{kV}$ de (maxinum) powr supply. This supply then feeds in parallel thres siparate. modulators (charging chokes, pulse forming networks a1:! thyratron switches), one at each of the klystrons. Each klystron has a 12:1 step-up pulse transformer in its cath. ode oil tank. The pulse transformer delivers upwards to $300 \mathrm{kV}$, which means upwards to $30 \mathrm{MW}$ plus of peak RF power from each klystron. Individual modulators and klystrons may be taken off-line by means of disconnect bars in the high voltage power supply distribution hox.

The RF drive circuit, shown in Fig. 3, starts with a $2856 \mathrm{MHz}, 5 \mathrm{~mW}$ VCO that drives a $1 \mathrm{~kW}$ peak. Mlicon/MPD, RF amplifier. The latter contains of a solid state, $1 \mathrm{~W} c \mathrm{cw}$ preamplifier and three cascaded Einat $8847 \mathrm{~A}$ planar triodes that are operated class $C$ for $6 \mu \mathrm{s}$ at the $10 \mathrm{~Hz}$ rate, as shown in Fig. 5a. SLAC-built. mediun power ( $<2 \mathrm{~kW}$ peak), solid-state, varjable at ienuators $[i]$ are used at the input to each klystron. Microwave Applications Group's, analog, rotary-field, ferrite, phase shifiers are used at the inputs to klystrons 1 and 3 . which ar" driven by signals derived from the output of klystron 2. as done at BNL's light source. A motor driven altenua. tor and phase shifter are installed in the gun's high power input waveguide. In addition, the RF power level out of klystron 3 has been made subject to the beam energy, as monitored by a beam position monitor (BPM) located arter the first bend magnet at the end of the linac. The BPM signals are processed and fed through a leedback loop to drive attenuator 3; thus, beam energy fluctuations are damped. The other two klystrons are normally run saturated. When any klystron sees a refected signal greater than 3 MW (a VSWR of 2:1 at $30 \mathrm{MW}$ ), an interlock circuit switches to maximum attenuation to drop the klyst ron output power. RF processing of the DLWG's and loads is accomplished by varying the appropriate attenuators. By using proper combinations of the three phase shifters. the gun phase can be adjusted relative to the whole linac and each DLWG can be phased separately relative to the gun Of course, field changes in the alpha magnel produce phas drifts, which can be corrected for, too. The gun resonant frequency is fine tuned by changing its water temperature By adjusting the gun input RF power and the gun curreut. by way of the gun filament temperature, bearn loading can be optimized as shown in Fig. 5e.

The circulator shown in Fig. 3 and $\$$ is not a vacuum item, so it is isolated by windows from the otherwise vacuum rectangular waveguide. It is pressurized with dry nitrogen to 30 psig, which is marginal for 3 to $4 \mathrm{MW}$. Sulphur hexafloride was not used because of possible, irreversible. linac section damage from a catastroplic vacuum failur. during operation. It is not clear the circulator is necessary. so its removal is being considered, especially if and when lests of the gun at higher RF power are performed.

The linac vacuum system cont ains one $81 / 5$. one $201 / 5$ and nine $30 \mathrm{l} / \mathrm{s}$ ion pumps and four iouization gauge... at 

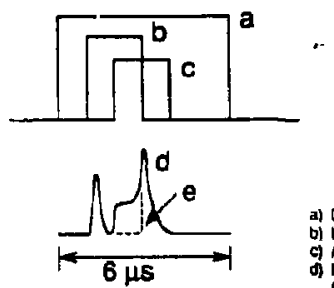

\section{a) Orne amplitiar RF ousput.}

b) Kystron AF eurpur.

c) Accolerator cection RF oumut.

d) Micowave guts redected RF whth the heater of (no baant).

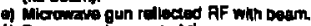

1) Baam anvelipe out of the qun.

of Eann cheppor putal

ii) Chopped beam mitereputes out of the lines.

i) Fing ou fif expand ed to ahow timing ka capture of the linac basm.

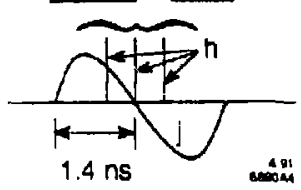

Figure 5. Linac and Bonster Ring Pulse, RF and Benm Tining.

show'l in Fig. 3. A beamline vacuum valve and waveguide window allow isolation of the gun vacuum from the linac when desired and a beamline valve at the end of the linac allows isolation of the linac. The ionization gauges operate interlocks that switch off the RF if the pressure goes too high. The linac has three different tempcrature water circuits; 1) $25^{\circ} \pm 5^{\circ} \mathrm{C}$ low conductivity water from the SLAC system for the klystrons, 2) $45^{\circ} \pm 0.1^{\circ} \mathrm{C}$ water for the DLWG's and 3) $35^{\circ}$ to $55^{\circ} \mathrm{C}$, adjustable but stable to $\pm<1^{\circ} \mathrm{C}$, water for the microwave gun, depending upon its operating parameters. The second system is self contained with a purnp, a heater, a heat exchanger (the heat is rejected to the SLAC system) and a mixing valve. It supplies $60 \mathrm{gpm}$ at 70 psig. The third system Laps of $<2 \mathrm{gpm}$ from the second system and puts it through a heater and/or a heat exchanger to get the desired temperature at tle gun, then returns it. Separate temperature sensors for the DLWG's and the gun work through two controllers to regulate the mixing valve and a beater, respectively:

\section{Booster Ring RF}

As shown in Fig. 1 and 6 , a second lower frequency RF system is part of the injector and SPEAR. The booster ring is 160 wavelengths in circumference and SPEAR is 280, so every seventh turn around the booster ring is synchronous with the same bucket in SPEAR on every fourtl turn. Three bunches from the linac are let into the booster at the right time, as seen in Fig. $5 \mathrm{~h}$ and $\mathrm{j}$, by synchronizing the chopper with the booster RF. The booster RF cavity is an early SPEAR cavity refurbished, the $358.54 \mathrm{MHz}$, $500 \mathrm{~kW}$ cw klystron is a PEP type tube tuned for the slightly different (atas!) SPEAR frequency and the control system is a carbon (or Xerox?) copy of PEP's [8] Since the booster ring is more stable if the RF accelerating fields are not so strong during the low energy part of the ramping cycle, the reference for the amplitude feedback circuit is ramped along with the synchrotron, as shown in Fig. 6.

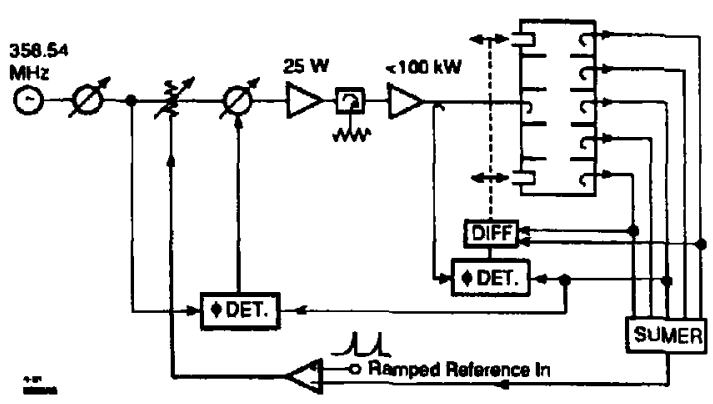

Figure 6. Injector Booster Ring RF Circuit.

\section{Operations}

The microwave gun, alpha magnet and chopper came on-line without much difficulty, although numerous hours were spent studying and characterizing the preinjector. The linac high power RF networks RF processed in less than 40 hours initially and now pump down in less than a day, if let up to nitrogen. It takes several days, and a hot nitrogen bake of the RF loads may be required, if the linac is let up to air. The modulators had a few high voltage growing pains, but they have settled down siuce. The chopper with its permanent magnets is designed to limit excessive beam transmission (potentially 1000 times the design value), but under poor set-up conditions for the GTL (Fig. 2) or unusual failure modes for the chopper (eg., slow rise time) the linac is slut off by average current monitoring circuits. In general the RF circuits for the injector are not required to be vltra stable or critically adjusted. So the broad optimums often encountered for the GTL settings in particular have not been a fundamental limitation.

\section{References}

[1] H. Wiedemann et al., "3 GeV Injector Synchrolron for SPEAR," Proc. 1991 PAC, San Francisco, CA.

[2] M. Borland et al., "Design and Performance of the Traveling-Wave Beam Chopper for the SSIRL Injector," Proc 1991 PAC, San Francisco, CA

[3] R. Hettel et al, "Triggers and Timing System for the SSRL 3 GeV Injector," Proc. 1991 PAC, San Francisco, CA.

[4] M. Borland, $P h D$ thesis, Stanford University. (1991).

[5] M. Borland et al., "Performance of the $2 \mathrm{MeV}$ Microwave Gun for the SSRL $150 \mathrm{MeV}$ Linar." Proc. 1990 Linac ConI., 761-3, Albuquerque. NiI

[6] Tanabe et al., SLAC-PUB-5054, (Aug. 1989).

[7] H. Schwarz, IEEE Trans., NS-32, 1847-51, (Oct 1985).

[8] J.-L. Pellegrin et al., IEEE Trans., NS-28. 2320 2. (Jun 1981). 